\section{PS-369 TIMING OF VOIDING CYSTOURETHROGRAM IN THE INVESTIGATION OF URINARY TRACT INFECTIONS IN CHILDREN: A SYSTEMATIC REVIEW AND META-ANALYSIS}

${ }^{1}$ A Merglen, ${ }^{2} \mathrm{M}$ Katz-Lavigne, ${ }^{3} \mathrm{~T}$ Agoritsas, ${ }^{4} \mathrm{SA}$ Asner, ${ }^{2} \mathrm{EM}$ Uleryk, ${ }^{2} \mathrm{~J}$ Friedman, ${ }^{2} \mathrm{P}$ Parkin, ${ }^{2}$ E Cohen, ${ }^{2} S$ Mahant. ${ }^{1}$ Paediatrics, Geneva University Hospitals, Geneva, Switzerland; ${ }^{2}$ Paediatrics, The Hospital for Sick Children, Toronto, Canada; ${ }^{3}$ Health Sciences, McMaster University, Hamilton, Canada; ${ }^{4}$ Paediatrics, CHUV, Lausanne, Switzerland

\subsection{6/archdischild-2014-307384.667}

Background Voiding cystourethrogram (VCUG) is considered the gold standard for detecting vesicoureteral reflux (VUR). Optimal timing of VCUG after urinary tract infections (UTI) is controversial. Single studies have compared rates of VUR according to timing of VCUG, but included a limited number of patients and failed to reach definitive conclusions.

Objective We conducted a systematic review and meta-analysis of studies that compared rates of VUR when VCUG was performed early versus late in the investigation of UTI.

Methods We searched MEDLINE, EMBASE, CINHAL and CENTRAL. We included studies that compared the rates of VUR between early versus late-performed VCUG in children diagnosed with UTI. We used the threshold of early vs. late reported by the authors: most commonly at 7 days, but no later than 14 days. Studies that focused on patients with previously diagnosed VUR or other urogenital pathology were excluded. Pairs of reviewers independently screened potentially eligible articles, extracted data and assessed quality of the evidence according to the GRADE framework.

Results Of the 1526 studies reviewed, 7 studies with 1623 patients were included ( 2 prospective and 5 retrospective cohort studies). Meta-analysis showed no increased risk of VUR in early versus late performed VCUG (Odds ratio 0.99 , 95\% CI 0.77 to 1.27 , corresponding to a risk difference of $0.00,95 \%$ CI -0.05 to $\left.0.04, \mathrm{I}^{2}=0 \%\right)$. The overall quality of the evidence was moderate.

Conclusions In children diagnosed with UTI, there is no significant difference in rates of VUR when VCUG is performed early rather than later.

\section{PS-370 OUTCOME OF ANTIMICROBIAL THERAPY OF PAEDIATRIC URINARY TRACT INFECTIONS CAUSED BY EXTENDED-SPECTRUM B-LACTAMASE-PRODUCING ENTEROBACTERIACEAE}

${ }^{1}$ EH Choi, ${ }^{2} \mathrm{HS}$ Kim, ${ }^{1} \mathrm{BJ}$ Lee, ${ }^{1} \mathrm{HJ}$ Lee, ${ }^{1} \mathrm{HG}$ Kang, ${ }^{1} \mathrm{IS} \mathrm{Ha},{ }^{1} \mathrm{HI}$ Cheong. ${ }^{1}$ Pediatrics, Seoul National University College of Medicine, Seoul, Korea; ${ }^{2}$ Pediatrics, Dongguk University IIsan Hospital Dongguk University School of Medicine, Goyang, Korea

\subsection{6/archdischild-2014-307384.668}

Purpose The purpose of this study was to compare the outcome of carbapenem versus non-carbapenem antimicrobial therapy for paediatric urinary tract infections (UTIs) caused by extendedspectrum $\beta$-lactamase (ESBL) producing Enterobacteriaceae.

Methods From 2006 to 2011, 42 episodes of UTI caused by ESBL-producing Enterobacteriaceae were diagnosed at Seoul National University Children's Hospital. Patients were grouped according to the antimicrobials they received into a carbapenem group and a non-carbapenem group. Medical records were retrospectively reviewed to assess treatment outcome, time todefervescence after initiation of treatment, and relapse rate.
Results There were 36 children with 42 episodes of UTI caused by ESBL-producing Enterobacteriaceae. Twenty-seven cases (64\%) had an underlying urologic disease, 28 (67\%) cases were caused by Escherichia coli, and 14 (33\%) cases were caused by Klebsiella pneumoniae. Four (10\%) cases were treated with carbapenem, 23 cases $(55 \%)$ were treated with non-carbapenem, and 15 (36\%) cases were treated by switching from a carbapenem to a non-carbapenem and vice versa. There was no treatment failure at the time of antimicrobial discontinuation. Between the carbapenem and the non-carbapenem treatment groups, there were no significant differences in bacterial aetiology $(\mathrm{p}=0.59)$, time todefervescence after the initiation of antimicrobials $(\mathrm{p}=0.28)$, and relapse rate $(\mathrm{p}=$ $0.50)$. In vitro susceptibility to non-carbapenem antimicrobials did not affect the time todefervescence after the initiation of antimicrobial treatment, and the relapse rate in the non-carbapenem group.

Conclusion This study found no significant difference in the treatment outcome between paediatric patients treated with carbapenem and those treated with non-carbapenem antimicrobials for UTI caused by ESBL-producing Enterobacteriaceae. Therefore, the initially administered non-carbapenem therapy can be maintained in UTI patients showing clinical improvement.

\section{PS-371 RESPIRATORY VIRUSES MIGHT INCREASE THE RISK OF ACQUIRING URINARY TRACT INFECTIONS}

M Hendaus, A Al-Hammadi, M Khalifa, E Muneer. Pediatrics, Hamad General Hospital, Doha, Qatar

\subsection{6/archdischild-2014-307384.669}

Background Clinicians often express concerns of serious bacterial infection when facing a child with acute bronchiolitis.

Aims The goals of this study were 1) to estimate the prevalence of urinary tract infection (UTI) in infants and children with bronchiolitis, and 2) to evaluate the effect of demographic, clinical characteristics and other related factors on the prevalence rates of UTI.

Materials and methods A descriptive and retrospective study was conducted at Hamad Medical Corporation, the only tertiary medical centre in the State of Qatar. Patients ages 0 to 36 months hospitalised with acute bronchiolitis from January 2010 to December 2012 were included in the study. The following data were collected: age at diagnosis, gestational age, nationality, sex, Direct Fluorescent Antibodies (DFA) and urine culture results. For the purpose of data analysis, we divided the patient population into three categories: RSV positive, RSV negative and other than RSV (influenza, Para influenza, parechovirus, etc).

Results 836 paediatric patients with acute bronchiolitis were admitted in paediatrics department ward unit. The mean age at diagnosis was $3.61 \pm 3.56$ months ranged from 0.33 to 34 months. There were $39.2 \%$ girls, 68.8\% boys; 61.4\% Qatari and $38.6 \%$ were Non-Qatari nationals. The percentage of UTI was significantly higher among other than RSV (30/224; 13.4\%) compared to RSV-positive $(11 / 224 ; 4.9 \%)$ and RSV-negative (8/ $100 ; 8 \%) ; \mathrm{p}=0.007$.

Conclusions Infants and young children with might have a higher risk of UTI if presented with bronchiolitis triggered by a respiratory virus other than RSV. 


\section{PS-372 DIFFUSE FATTY INFILTRATION IN STRESS-RELATED THYMIC INVOLUTION: AN INDEPENDENT FINDING FOR PAEDIATRIC INFECTION}

${ }^{1} \mathrm{M}$ Taweevisit, ${ }^{2} \mathrm{P}$ Thorner. ${ }^{1}$ Pathology, Chulalongkorn University, Bangkok, Thailand; 2 Pathology and Laboratory Medicine, University of Toronto, Toronto, Canada

10.1136/archdischild-2014-307384.670

Background and aims Fatty infiltration is known as a physiologic hallmark of thymic involution, starting at puberty (agerelated involution). However, the knowledge of fatty infiltration in thymuses whose paediatric patients suffering from acute illness (stress-related involution) has never been studied. The purpose of this work was to evaluate the frequency and degree of fatty infiltration and to correlate the degree of fatty infiltration with the severity of involution in thymic tissues belonging paediatric patients who died from various causes of illness.

Methods Thymic tissues from paediatric autopsy series were collected and processed for histopathologic examination. The degree of fatty infiltration was divided semi-quantitatively as absence, minimal (<5\%), occasional (5-50\%) and diffuse $(>50 \%)$. The severity of thymic involution was graded as 1 (resting state), 2 (more thymic lobule separation) and 3 (advanced stromal fibrosis).

Results Fatty infiltration (Figure 1) was found 36/130 cases $(28 \%)$ and tended to accumulate in grade 3 thymic involution ( $p$ $=0.01)$. In most cases, the degree was minimal (11 cases; 9\%) or occasional (18 cases; $14 \%$ ) and no statistical correlation with any clinical information. There were 7 cases (5\%) showing diffuse fatty infiltration and all of them died from infection (bronchopneumonia $=3$, meningitis $=2$, acute myocarditis with abscesses $=1$, acute pyelonephritis $=1$ ), regardless of specific organism, patient age or duration of illness.

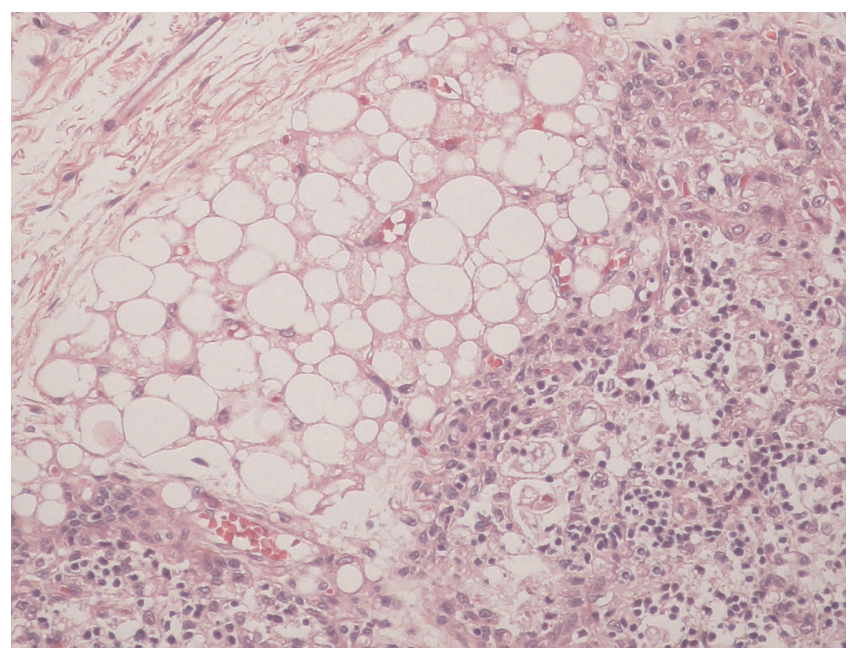

Abstract PS-372 Figure 1 Thymic fatty infiltration.

Conclusion In stress-related thymic involution, the frequency of diffuse fatty infiltration was rare, but such change was an independent finding associated with paediatric infection.

\section{PS-373 OPTIMISING A DIAGNOSTIC ALGORYTHM IN PATIENS PRESENTING FOR ADENOPATHY IN PRIMARY CARE SETTINGS}

R Bogdan, M Oros, C Codleanu, C Radu, O Dedu, A Dumineca, C Momirla. Pediatrics, Medicover Clinic, Bucharest, Romania
Aim To describe the etiologic spectrum, clinical characteristics and evaluate the adequateness of the tests performed in order to establish diagnosis of adenopathies in children.

Materials and method Our group analysed 279 children with adenopathies consulted in the primary care paediatric clinic in a 4 years period 2009-2013. There were noted: the presence and localization of the adenopathies, the diagnosis, the tests performed and the outcome.

Results Out of the 279 cases, 106 represented unspecific multiple adenopathies following past diseases and had no clinical significance. 77 cases were confirmed to be viral unspecific infections of the upper respiratory system, 16 cases were confirmed as Infectious mononucleosis, 22 cases were Acute bacterial Tonsilitis and 11 were confirmed as Group A Streptococcus Tonsilitis. Further investigation of cases with large adenopathies of unknown origin revealed less common etiologies as Toxocara and Toxoplasma infections. There was 1 case of Hodgkin lymphoma easily diagnosed based on clinical characteristics, confirmed and treated in the oncology hospital. 7 cases presented with typical left axilar satellite adenopathy following BCG vaccination. The history and clinical signs suggested the diagnosis in all the cases. In 27 of the cases with unique large adenopathies the parents refused further diagnostic tests.

Conclusions A complete history and a meticulous clinical examination represented the most important steps in establishing the etiologic diagnosis of the adenopathies in children. Specific blood tests and other investigations must be used with caution, in order to avoid unnecessary painful diagnostic procedures and unjustified costs.

\section{PS-374 A COMPARISON OF TREATMENT AT HOME OR IN HOSPITAL FOR MODERATE/SEVERE CELLULITIS IN CHILDREN}

${ }^{1}$ SM Hopper, ${ }^{2}$ LF Ibrahim, ${ }^{1} \mathrm{FE}$ Babl, ${ }^{3} \mathrm{PA}$ Bryant. 'Emergency Department, The Royal Children's Hospital and Murdoch Children's Research Institute, Parkville Melbourne, Australia; ${ }^{2}$ RCH@Home, The Royal Children's Hospital, Parkville Melbourne, Australia; ${ }^{3} \mathrm{R}$ CH@Home Infectious Diseases Unit, The Royal Children's Hospital and Murdoch Children's Research Institute, Parkville Melbourne, Australia

\subsection{6/archdischild-2014-307384.672}

Background and aims Adults with cellulitis are commonly receive IV antibiotics via hospital-in-the-home (HITH). Children are usually admitted to hospital. Royal Children's Hospital (RCH) HITH and offers once daily IV ceftriaxone for cellulitis. Concerns remain for some physicians about its anti-staphylococcal activity. We aim to compare the clinical features and outcomes of patients with cellulitis admitted to hospital with IV flucloxacillin to those treated via HITH with IV ceftriaxone.

Methods A retrospective chart review of patients with cellulitis treated with IV antibiotics. Exclusions- complicated cellulitis (abscess, orbital cellulitis, post-operative cellulitis, bites and immunosuppression). Demographics, clinical and microbiological features, antibiotic management and outcomes are related to two groups: inpatients treated with IV flucloxacillin and HITH patients treated with IV ceftriaxone.

Results Over 17 months (2012-2014), 745 children presented to ED with cellulitis: 353 (47\%) received IV antibiotics; 169 were excluded (complicated cellulitis, comorbidities, misdiagnosis or miscoding), leaving 184. 47 (26\%) were admitted to HITH and 137 (74\%) were admitted as inpatients. Initial treatment was IV ceftriaxone in $41(87 \%)$ of HITH patients and IV flucloxacillin in 103 (75\%) of inpatients. 